\title{
MODEL-THEORETIC CHARACTERIZATION OF INTUITIONISTIC PROPOSITIONAL FORMULAS
}

\author{
GRIGORY K. OLKHOVIKOV
}

Department of Philosophy, Ural Federal University

\begin{abstract}
Notions of $k$-asimulation and asimulation are introduced as asymmetric counterparts to $k$-bisimulation and bisimulation, respectively. It is proved that a first-order formula is equivalent to a standard translation of an intuitionistic propositional formula iff it is invariant with respect to $k$-asimulations for some $k$, and then that a first-order formula is equivalent to a standard translation of an intuitionistic propositional formula iff it is invariant with respect to asimulations. Finally, it is proved that a first-order formula is equivalent to a standard translation of an intuitionistic propositional formula over the class of intuitionistic Kripke models iff it is invariant with respect to asimulations between intuitionistic models.
\end{abstract}

\$1. Introduction. Van Benthem's well-known modal characterization theorem (Theorem 4.9 below) states that a first-order formula is equivalent to a standard translation of a modal propositional formula iff it is invariant with respect to bisimulations. There is also a weaker 'parametrized' version of this result stating that a first-order formula is equivalent to a standard translation of a modal propositional formula iff this formula is invariant with respect to $k$-bisimulations for some $k$. Although both results yield a convenient model-theoretical technique distinguishing 'modal' first-order formulas from 'nonmodal' ones, van Benthem's characterization theorem, unlike its parametrized version, also isolates a single property defining expressive powers of modal propositional logic and thus gives us an important insight into its nature when this logic is viewed as a fragment of first-order logic.

Given that the view of intuitionistic logic as a fragment of modal propositional logic has a long and established tradition dating back to Tarski-Gödel translation of this logic into $S 4$, it was natural to ask whether one can add to the bisimulation invariance some extra condition to get a criterion distinguishing 'intuitionistic propositional' first-order formulas from nonintuitionistic ones. As was shown by Visser et al. (1995), bisimulation invariance plus a form of monotonicity with respect to the accessibility relation 'almost' gives such a criterion: i. e., it tells intuitionistic propositional first-order formulas from nonintuitionistic ones over the set of intuitionistic models. It was not known, however, how to remove this restriction and provide a criterion distinguishing intuitionistic propositional first-order formulas from nonintuitionistic ones in the general case.

In this paper, we take a different strategy and amend the notion of bisimulation itself rather than adding new conditions to it. In this way, we get a notion of asimulation and we show that asimulation invariance allows one to tell intuitionistic propositional first-order formulas from nonintuitionistic ones in the general case, thus providing a criterion for the

Received: October 2, 2012. 
full unrestricted logical equivalence in the style of van Benthem's original modal characterization theorem. Then we show that a slight modification of asimulation invariance also gives us an essentially new form of the restricted equivalence criterion in the style of Visser et al.

The layout of the paper is as follows. Starting from some notational conventions and preliminary remarks in Section $\$ 2$, we then move on to the proof of a 'parametrized' version of model-theoretic characterization of intuitionistic propositional logic in Section $\S 3$ and finally prove the full unparametrized counterpart to van Benthem's characterization theorem for intuitionistic propositional logic in Section $\$ 4$. From this latter result we derive a characterization of equivalence of a first-order formula to a standard translation of intuitionistic formula over the class of intuitionistic models. This latter result is of interest, given that, unlike in the case of modal propositional logic, not every first-order model can be treated as an intended model of intuitionistic propositional logic. Finally, in Section $\$ 5$ we sum up and announce some further results obtained in this direction.

\$2. Preliminaries. A formula is a formula of classical predicate logic without identity whose predicate letters are in vocabulary $\Sigma=\left\{R^{2}, P_{1}^{1}, \ldots P_{n}^{1}, \ldots\right\}$. We assume $\{\perp, \rightarrow$, $\vee, \wedge, \forall, \exists\}$ as the set of basic connectives and quantifiers for this variant of classical firstorder language. A model is a model of this logic, that is to say, an ordered pair of a domain and an interpretation function assigning the extensions to predicate constants from a subset of $\Sigma$. We refer to formulas with lowercase Greek letters $\varphi, \psi$, and $\chi$, and to sets of formulas with uppercase Greek letters $\Gamma$ and $\Delta$. If $\varphi$ is a formula, then we associate with it the following finite vocabulary $\Sigma_{\varphi} \subseteq \Sigma$ such that $\Sigma_{\varphi}=\left\{R^{2}\right\} \cup\left\{P_{i} \mid P_{i}\right.$ occurs in $\left.\varphi\right\}$. Thus, if $\varphi$ contains an occurrence of $R$, then $\Sigma_{\varphi}$ is the smallest signature/vocabulary such that $\varphi$ can be expressed over it. More generally, we refer with $\Theta$ to an arbitrary subset of $\Sigma$ such that $R \in \Theta$. If $\psi$ is a formula and every predicate letter occurring in $\psi$ is in $\Theta$, then we call $\psi$ a $\Theta$-formula.

We refer to sequence $x_{1}, \ldots, x_{n}$ of any objects as $\bar{x}_{n}$. We identify a sequence consisting of a single element with this element. If all free variables of a formula $\varphi$ (formulas in $\Gamma$ ) coincide with a variable $x$, we write $\varphi(x)(\Gamma(x))$.

For a binary relation $S$ and any objects $s, t$ we abbreviate the fact that $s+\wedge t S s$ by $s \stackrel{\leftrightarrow}{S} t$

We use the following notation for models of classical predicate logic:

$$
M=\langle U, \imath\rangle, M_{1}=\left\langle U_{1}, \imath_{1}\right\rangle, M_{2}=\left\langle U_{2}, \imath_{2}\right\rangle, \ldots, M^{\prime}=\left\langle U^{\prime}, \imath^{\prime}\right\rangle, M^{\prime \prime}=\left\langle U^{\prime \prime}, \imath^{\prime \prime}\right\rangle, \ldots,
$$

where the first element of a model is its domain and the second element is its interpretation of predicate letters. If $k \in \mathbb{N}$ then we write $R_{k}$ as an abbreviation for $l_{k}(R)$. If $a \in U$ then we say that $(M, a)$ is a pointed model. Further, we say that $\varphi(x)$ is true at $(M, a)$ and write $M, a \models \varphi(x)$ iff for any variable assignment $\alpha$ in $M$ such that $\alpha(x)=a$ we have $M, \alpha \models \varphi(x)$. It follows from this convention that the truth of a formula $\varphi(x)$ at a pointed model is to some extent independent from the choice of its only free variable. Moreover, for $k \in \mathbb{N}$ we will sometimes write $a \models_{k} \varphi(x)$ instead of $M_{k}, a \models \varphi(x)$.

An intuitionistic formula is a formula of intuitionistic propositional logic, where $\{\perp, \rightarrow$, $\vee, \wedge\}$ is the set of basic connectives and $\left\{p_{n} \mid n \in \mathbb{N}\right\}$ is the set of propositional letters. We refer to intuitionistic formulas with letters $I, J, K$, possibly with primes or subscripts. We assume a standard Kripke semantics for intuitionistic propositional logic.

If $x$ is an individual variable in a first-order language, then by standard $x$-translation of intuitionistic formulas into classical first-order formulas we mean the following map $S T$ 
defined by induction on the complexity of the corresponding intuitionistic formula. The induction goes as follows:

$$
\begin{aligned}
S T\left(p_{n}, x\right) & =P_{n}(x) \\
S T(\perp, x) & =\perp ; \\
S T(I \wedge J, x) & =S T(I, x) \wedge S T(J, x) ; \\
S T(I \vee J, x) & =S T(I, x) \vee S T(J, x) ; \\
S T(I \rightarrow J, x) & =\forall y(R(x, y) \rightarrow(S T(I, y) \rightarrow S T(J, y))) .
\end{aligned}
$$

In the last line it is assumed that $y$ does not occur in either $S T(I, x)$ or $S T(J, x)$.

By degree of a classical first-order formula we mean the greatest number of nested quantifiers occurring in it. Degree of a formula $\varphi$ is denoted by $r(\varphi)$. Its formal definition by induction on the complexity of $\varphi$ goes as follows:

$$
\begin{aligned}
r(\perp) & =r(\varphi)=0 & & \text { for atomic } \varphi \\
r(\varphi \circ \psi) & =\max (r(\varphi), r(\psi)) & & \text { for } \circ \in\{\wedge, \vee, \rightarrow\} \\
r(Q \times \varphi) & =r(\varphi)+1 & & \text { for } Q \in\{\forall, \exists\}
\end{aligned}
$$

For $k \in \mathbb{N}$, we say that $\Theta$-formula $\varphi(x)$ such that $r(\varphi) \leq k$ is a $(\Theta, x, k)$-formula.

§3. A parametrized version of the main result. We start with the introduction of an 'intuitionistic' counterpart of $k$-bisimulation. Its formal definition looks like this:

DeFINITION 3.1. Let $\left(M_{1}, t\right),\left(M_{2}, u\right)$ be two pointed $\Theta$-models. A binary relation $A$ is called $\left\langle\left(M_{1}, t\right),\left(M_{2}, u\right)\right\rangle_{k}$-asimulation iff for any $i, j \in\{1,2\}$, any $\bar{a}_{m}, a \in U_{i}, \bar{b}_{m}, b, d \in$ $U_{j}$, any unary predicate letter $P \in \Theta$, the following conditions hold:

$$
A \subseteq \bigcup_{n>0}\left(\left(U_{1}^{n} \times U_{2}^{n}\right) \cup\left(U_{2}^{n} \times U_{1}^{n}\right)\right)
$$

$t A u$

$$
\begin{aligned}
& \left(\left(\bar{a}_{m}, a\right) A\left(\bar{b}_{m}, b\right) \wedge a \models_{i} P(x)\right) \Rightarrow b \models_{j} P(x) \\
& \left(\left(\bar{a}_{m}, a\right) A\left(\bar{b}_{m}, b\right) \wedge b R_{j} d \wedge m<k\right) \Rightarrow \\
& \quad \Rightarrow \exists c \in U_{i}\left(a R_{i} c \wedge\left(\bar{a}_{m}, a, c\right) \stackrel{\leftrightarrow}{A}\left(\bar{b}_{m}, b, d\right)\right)
\end{aligned}
$$

It is perhaps best to explain the peculiarities of the idea of asimulation by informally dwelling on its differences from a much more standard notion of bisimulation (which is also defined below—see Definition 4.8). Unlike in bisimulation, where one basically tries to capture the coincidence of sets of propositional modal formulas true at two different states of Kripke models (this works perfectly only for saturated models, but we shall neglect it for the time being), in asimulation we try to capture the inclusion of the set of intuitionistic propositional formulas true at the 'left' state into the corresponding set for the 'right' state. This necessitates that asimulation does not have to be symmetrical and that, with asimulation, direction matters. This link with inclusion rather than coincidence also dismisses the need for the (forth) clause present in definition of bisimulation. To grasp this point one should remember that implication is virtually the only 'modal' connective of intuitionistic propositional logic, so one has to care about states accessible from a given state only as long as those states are able to falsify some implications claimed for the 
given state. Now, whatever counterexamples to intuitionistic implications may happen to be present in the states accessible from the 'left' state, these counterexamples do not need to be present among the successors of the 'right' state, since the implications in question might be actually true in this 'right' state - and this is why we do not need the (forth) clause. However, the analogue of (back) clause is still necessary and one even has to make it rather strong, since your typical counterexample to an intuitionistic implication will involve both the truth of antecedent and the falsehood of consequent of the implication at a given successor. Thus in order to ensure right-to-left transition of counterexamples to intuitionistic implications we have to demand not only inclusion but coincidence of sets of formulas true in the corresponding successors of two given states. This is why a symmetrical asimulation link is present in the consequent of condition (p-step).

The notion of $k$-asimulation is only different from asimulation simpliciter in that we introduce $k$ as the bound on the number of transitions from a given pair of states to a pair of their successors. We ensure this bound by making the relation of asimulation 'remember' the previous transitions, so that the transition is made to a path including all the previously visited states along with the chosen successors rather than simply to a pair of successors (in fact, 'p' in the labels of conditions in the above definition is supposed to stand for 'path').

EXAmple 3.2. Consider two $\left\{R^{2}, P^{1}\right\}$-models $M_{1}$ and $M_{2}$ such that $U_{1}=\{a, b, c\}$, $R_{1}=\{(a, b),(a, c)\}, \iota_{1}(P)=\{c\}$, and $U_{2}=\{d, e\}, R_{2}=\{(d, e)\}, \iota_{2}(P)=\{d\}$. Then binary relation $A$ (see Figure 1) such that a $A d,(d, e) A(a, b)$ and $(a, b) A(d, e)$ is an $\left\langle\left(M_{1}, a\right),\left(M_{2}, d\right)\right\rangle_{k}$-asimulation for every $k \in \mathbb{N}$.

We will only need the following lemma as a stepping stone towards Corollary 3.6. Its rather involved formulation is due to the fact that because of condition (p-step) we have to keep an eye on links between states running in both directions even while establishing a one-way connection.

LEMmA 3.3. Let $\varphi(x)=S T(I, x)$ for some intuitionistic formula $I$, and let $r(\varphi)=k$. Let $\Sigma_{\varphi} \subseteq \Theta$, let $\left(M_{1}, t\right),\left(M_{2}, u\right)$ be two pointed $\Theta$-models, and let $A$ be an $\left\langle\left(M_{1}, t\right),\left(M_{2}, u\right)\right\rangle_{l}$-asimulation. Then

$$
\left(\left(\bar{a}_{m}, a\right) A\left(\bar{b}_{m}, b\right) \wedge m+k \leq l \wedge a \models_{i} \varphi(x)\right) \Rightarrow b \models_{j} \varphi(x),
$$

for all $i, j \in\{1,2\},\left(\bar{a}_{m}, a\right) \in U_{i}^{m+1}$, and $\left(\bar{b}_{m}, b\right) \in U_{j}^{m+1}$.

Proof. We proceed by induction on the complexity of $I$. In what follows we will abbreviate the induction hypothesis by $\mathrm{IH}$.

Basis. Let $I=p_{n}$. Then $\varphi(x)=P_{n}(x)$ and the lemma follows from (p-base) together with the assumption that $\Sigma_{\varphi} \subseteq \Theta$.

The case $i=\perp$ is obvious, as $\perp$ never holds.

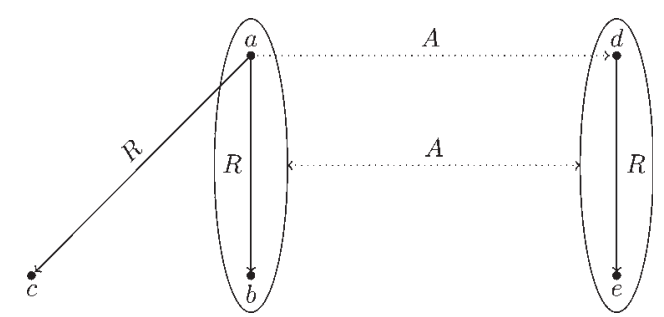

Fig. 1. Relation of $k$-asimulation between $M_{1}$ and $M_{2}$ from Example 3.2. 
Induction step.

The first two cases basically reduce to application of $\mathrm{IH}$; however, we spell them out in full detail below:

Case 1. Let $I=J \wedge K$. Then $\varphi(x)=S T(J, x) \wedge S T(K, x)$ and we reason as follows:

$$
\begin{array}{ll}
\left(\bar{a}_{m}, a\right) A\left(\bar{b}_{m}, b\right) & \text { (premise) } \\
a \models_{i} S T(J, x) \wedge S T(K, x) & \text { (premise) } \\
m+r(S T(J, x) \wedge S T(K, x)) \leq l & \text { (premise) } \\
r(S T(J, x)) \leq r(S T(J, x) \wedge S T(K, x)) & \text { (by df of } r) \\
r(S T(K, x)) \leq r(S T(J, x) \wedge S T(K, x)) & \text { (by df of } r) \\
a \models_{i} S T(J, x) & \text { (from (2)) } \\
a \models_{i} S T(K, x) & \text { (from (2)) } \\
m+r(S T(J, x)) \leq l & \text { (from (3) and (4)) } \\
m+r(S T(K, x)) \leq l & \text { (from (3) and (5)) } \\
b \models_{j} S T(J, x) & \text { (from (1), (6), and (8) by IH) } \\
b \models_{j} S T(K, x) & \text { (from (1), (7), and (9) by IH) } \\
b \models_{j} S T(J, x) \wedge S T(K, x) & \text { (from (10) and (11)) }
\end{array}
$$

Case 2. Let $I=J \vee K$. Then $\varphi(x)=S T(J, x) \vee S T(K, x)$ and we have then $a \models_{i}$ $S T(J, x) \vee S T(K, x)$. Assume, for convenience, that $a \models_{i} S T(J, x)$, the other case is similar. Then we reason as follows:

$$
\begin{array}{ll}
a \models_{i} S T(J, x) & \text { (premise) } \\
\left(\bar{a}_{m}, a\right) A\left(\bar{b}_{m}, b\right) & \text { (premise) } \\
m+r(S T(J, x) \vee S T(K, x)) \leq l & \text { (premise) } \\
r(S T(J, x)) \leq r(S T(J, x) \vee S T(K, x)) & \text { (by df of } r) \\
m+r(S T(J, x)) \leq l & \text { (from (15) and (16)) } \\
b \models_{j} S T(J, x) & \text { (from (13), (14), and (17) by IH) } \\
b \models_{j} S T(J, x) \vee S T(K, x) & \text { (from (18)) }
\end{array}
$$

Case 3. Let $I=J \rightarrow K$. Then

$$
\varphi(x)=\forall y(R(x, y) \rightarrow(S T(J, y) \rightarrow S T(K, y))) .
$$

Assume that:

$$
\begin{aligned}
& a \models_{i} \forall y(R(x, y) \rightarrow(S T(J, y) \rightarrow S T(K, y))) \\
& \left(\bar{a}_{m}, a\right) A\left(\bar{b}_{m}, b\right) \\
& m+r(\varphi(x)) \leq l
\end{aligned}
$$

Moreover, it follows from definition of $r$ that:

$$
\begin{aligned}
& r(\varphi(x)) \geq 1 \\
& r(S T(J, y)) \leq r(\varphi(x))-1 \\
& r(S T(K, y)) \leq r(\varphi(x))-1
\end{aligned}
$$


Now, consider an arbitrary $d \in U_{j}$ such that $b R_{j} d$. Since (22) and (23) clearly imply that $m<l$, it follows from (21) and (p-step) that one can choose a $c \in U_{i}$, such that:

$$
\begin{aligned}
& a R_{i} c \\
& \left(\bar{b}_{m}, b, d\right) A\left(\bar{a}_{m}, a, c\right) \\
& \left(\bar{a}_{m}, a, c\right) A\left(\bar{b}_{m}, b, d\right)
\end{aligned}
$$

So, we reason as follows:

$$
\begin{array}{ll}
d \models_{j} S T(J, y) & \text { (premise) } \\
m+1+r(S T(J, y)) \leq l & \text { (from (22) and (24)) } \\
m+1+r(S T(K, y)) \leq l & \text { (from (22) and (25)) } \\
c \models_{i} S T(J, y) & \text { (from (27), (29), (30) by IH) } \\
c \models_{i} S T(K, y) & \text { (from (20), (26), and (32)) } \\
d \models_{j} S T(K, y) & \text { (from (28), (33), (31) by IH) }
\end{array}
$$

Since $d$ was chosen to be an arbitrary $R_{j}$-successor of $b$, this means that

$$
b \models_{j} \forall y(R(x, y) \rightarrow(S T(J, y) \rightarrow S T(K, y))),
$$

and we are done.

We will now define the general notion of invariance; in its different variants it figures prominently in the main results of the present paper.

DEFINITION 3.4. Let $\alpha$ be a class of relations such that for any $A \in \alpha$ there is $a \Theta$ and there are $\Theta$-models $M_{1}$ and $M_{2}$ such that (p-type) holds. Then a formula $\varphi(x)$ is said to be invariant with respect to $\alpha$, iff for any $A \in \alpha$ for any corresponding $\Theta$-models $M_{1}$ and $M_{2}$, and for any $a \in U_{1}$ and $b \in U_{2}$ it is true that:

$$
\left(a A b \wedge a \models_{1} \varphi(x)\right) \Rightarrow b \models_{2} \varphi(x) .
$$

Thus, we say that $\varphi(x)$ is invariant with respect to $k$-asimulations, iff it is invariant with respect to the class of $k$-asimulations, and similarly for asimulations simpliciter and bisimulations to be defined in the next section.

EXAMPLE 3.5. Consider again the models $M_{1}$ and $M_{2}$ and the binary relation $A$ from Example 3.2. Formula $\exists y(R(x, y) \wedge P(y))$ is true at $\left(M_{1}, a\right)$, but not at $\left(M_{2}, d\right)$. So, since for every $k \in \mathbb{N}$ we know $A$ to be an $\left\langle\left(M_{1}, a\right),\left(M_{2}, d\right)\right\rangle_{k}$-asimulation, we get that there is no $k$ such that this formula is invariant with respect to $k$-asimulations.

The following corollary establishes an important special case of the 'only if' part of Theorem 3.12, which is our main aim in the present section:

COROLlary 3.6. If $\varphi(x)$ is a standard $x$-translation of an intuitionistic formula and $r(\varphi)=k$, then $\varphi(x)$ is invariant with respect to $k$-asimulations.

Corollary 3.6 immediately follows from Lemma 3.3 setting $i:=1, j:=2, m:=0$, and $l:=k$.

Before we state and prove the parametrized version of our main result, we need to mention a fact from the classical model theory of first-order logic.

LEMMA 3.7. For any finite predicate vocabulary $\Theta$, any variable $x$ and any natural $k$ there are, up to logical equivalence, only finitely many $(\Theta, x, k)$-formulas. 
This fact is proved as lemma 3.4 in Ebbinghaus et al. (1984, pp. 189-190). It allows us to collect logical equivalents of all standard translations of intuitionistic formulas which are true together with a given formula at some pointed model in a single formula which we will call a complete conjunction:

DeFINITION 3.8. Let $\varphi(x)$ be a formula. A conjunction of $\left(\Sigma_{\varphi}, x, k\right)$-formulas $\Psi(x)$ is called a complete $(\varphi, x, k)$-conjunction iff (1) every conjunct in $\Psi(x)$ is a standard $x$ translation of an intuitionistic formula; and (2) there is a pointed model $(M, a)$ such that $M, a \models \Psi(x) \wedge \varphi(x)$ and for any $\left(\Sigma_{\varphi}, x, k\right)$-formula $\psi(x)$, if $\psi(x)$ is a standard $x$ translation of an intuitionistic formula and $M, a \models \psi(x)$, then $\Psi(x) \models \psi(x)$.

The two following lemmas establish some straightforward properties of complete conjunctions:

LEMma 3.9. For any formula $\varphi(x)$, any natural $k$, any $\Theta$ such that $\Sigma_{\varphi} \subseteq \Theta$ and any pointed $\Theta$-model $(M, a)$ such that $M, a \models \varphi(x)$ there is a complete $(\varphi, x, k)$-conjunction $\Psi(x)$ such that $M, a \models \Psi(x) \wedge \varphi(x)$.

Proof. Let $\Gamma$ be the set of all $\left(\Sigma_{\varphi}, x, k\right)$-formulas that are standard $x$-translations of intuitionistic formulas true at $(M, a)$. This set is nonempty since $S T(\perp \rightarrow \perp, x)$ will be true at $(M, a)$. Due to Lemma 3.7, we can choose in this set a nonempty finite subset $\Gamma^{\prime}$ such that any formula from $\Gamma$ is logically equivalent to (and hence follows from) a formula in $\Gamma^{\prime}$. Therefore, every formula in $\Gamma$ follows from $\Lambda \Gamma^{\prime}$ and we also have $M, a \models \wedge \Gamma^{\prime}$, therefore, $\bigwedge \Gamma^{\prime}$ is a complete $(\varphi, x, k)$-conjunction.

LEMMA 3.10. For any formula $\varphi(x)$ and any natural $k$ there are, up to logical equivalence, only finitely many complete $(\varphi, x, k)$-conjunctions.

Proof. It suffices to observe that for any formula $\varphi(x)$ and any natural $k$, a complete $(\varphi, x, k)$-conjunction is a $\left(\Sigma_{\varphi}, x, k\right)$-formula. Our lemma then follows from Lemma 3.7.

Proceeding to the main result of the present section, we first establish the 'hard' rightto-left direction of Theorem 3.12 which we prove as a separate theorem:

THEOREM 3.11. Let $k$ be $r(\varphi(x))$ and let $\varphi(x)$ be invariant with respect to $k$-asimulations. Then $\varphi(x)$ is equivalent to a standard $x$-translation of an intuitionistic formula.

Proof. We may assume that both $\varphi(x)$ and $\neg \varphi(x)$ are satisfiable, since both $\perp$ and $T$ are obviously invariant with respect to $k$-asimulations and we have, for example, the following valid formulas:

$$
\perp \leftrightarrow S T(\perp, x), \top \leftrightarrow S T(\perp \rightarrow \perp, x) .
$$

We may also assume that there are two complete $(\varphi, x, k+2)$-conjunctions $\Psi(x), \Psi^{\prime}(x)$ such that $\Psi^{\prime}(x) \models \Psi(x)$, and both formulas $\Psi(x) \wedge \varphi(x)$ and $\Psi^{\prime}(x) \wedge \neg \varphi(x)$ are satisfiable.

For suppose otherwise. Then take the set of all complete $(\varphi, x, k+2)$-conjunctions $\Psi(x)$ such that the formula $\Psi(x) \wedge \varphi(x)$ is satisfiable. This set is nonempty, because $\varphi(x)$ is satisfiable, and by Lemma 3.9, it can be satisfied only together with some complete $(\varphi, x, k+2)$-conjunction. Now, using Lemma 3.10, choose in it a finite nonempty subset $\left\{\Psi_{i_{1}}(x) \ldots, \Psi_{i_{n}}(x)\right\}$ such that any complete $(\varphi, x, k+2)$-conjunction is equivalent to an element of this subset. We can show that $\varphi(x)$ is logically equivalent to $\Psi_{i_{1}}(x) \vee \ldots \vee$ 
$\Psi_{i_{n}}(x)$. In fact, if $M, a \models \varphi(x)$ then, by Lemma 3.9, at least one complete $(\varphi, x, k+2)$ conjunction is true at $(M, a)$ and therefore, its equivalent in $\left\{\Psi_{i_{1}}(x) \ldots, \Psi_{i_{n}}(x)\right\}$ is also true at $(M, a)$, and so, finally we have

$$
M, a \models \Psi_{i_{1}}(x) \vee \ldots \vee \Psi_{i_{n}}(x) .
$$

In the other direction, if $M, a \models \Psi_{i_{1}}(x) \vee \ldots \vee \Psi_{i_{n}}(x)$, then for some $1 \leq j \leq n$ we have $M, a \models \Psi_{i_{j}}(x)$. Then, since $\Psi_{i_{j}}(x) \models \Psi_{i_{j}}(x)$ and by the choice of $\Psi_{i_{j}}(x)$ the formula $\Psi_{i_{j}}(x) \wedge \varphi(x)$ is satisfiable, so, by our assumption, the formula $\Psi_{i_{j}}(x) \wedge \neg \varphi(x)$ must be unsatisfiable, and hence $\varphi(x)$ must follow from $\Psi_{i_{j}}(x)$. But in this case we will have $M, a \models \varphi(x)$ as well. So $\varphi(x)$ is logically equivalent to $\Psi_{i_{1}}(x) \vee \ldots, \vee \Psi_{i_{n}}(x)$ but the latter formula, being a disjunction of conjunctions of standard $x$-translations of intuitionistic formulas, is itself a standard $x$-translation of an intuitionistic formula, and so we are done.

If, on the other hand, one can take two complete $(\varphi, x, k+2)$-conjunctions $\Psi(x), \Psi^{\prime}(x)$ such that $\Psi^{\prime}(x) \models \Psi(x)$, and formulas $\Psi(x) \wedge \varphi(x)$ and $\Psi^{\prime}(x) \wedge \neg \varphi(x)$ are satisfiable, we reason as follows. Take a pointed $\Sigma_{\varphi}$-model $\left(M_{1}, t\right)$ such that $t \models_{1} \Psi(x) \wedge \varphi(x)$ and for any $\left(\Sigma_{\varphi}, x, k+2\right)$-formula $\psi(x)$, if $\psi(x)$ is a standard $x$-translation of an intuitionistic formula true at $\left(M_{1}, t\right)$, then $\psi(x)$ follows from $\Psi(x)$, and take any pointed model $\left(M_{2}, u\right)$ such that $u \models_{2} \Psi^{\prime}(x) \wedge \neg \varphi(x)$.

We can construct an $\left\langle\left(M_{1}, t\right),\left(M_{2}, u\right)\right\rangle_{k}$-asimulation and thus obtain a contradiction in the following way. First, let us adopt a new piece of notation. If $i, j \in\{1,2\}$ and $x$ is a variable, then for any $g \in U_{i}$ and $h \in U_{j}$, we denote the fact that all $\left(\Sigma_{\varphi}, x, k\right)$ formulas that are standard $x$-translations of intuitionistic formulas true at $\left(M_{i}, g\right)$, are also true at $\left(M_{j}, h\right)$ as follows:

$$
\left(M_{i}, g\right) \leq_{k}\left(M_{j}, h\right) .
$$

Now, let $i, j \in\{1,2\}$ and let $\left(\bar{a}_{m}, a\right) \in U_{i}^{m+1},\left(\bar{b}_{m}, b\right) \in U_{j}^{m+1}$. Then

$$
\left(\bar{a}_{m}, a\right) A\left(\bar{b}_{m}, b\right) \Leftrightarrow\left(m \leq k \wedge\left(M_{i}, a\right) \leq_{k-m+2}\left(M_{j}, b\right)\right) .
$$

In particular, for $a \in U_{i}$ and $b \in U_{j}$ we have $a A b$ iff $\left(M_{i}, a\right) \leq_{k+2}\left(M_{j}, b\right)$.

It is clear that $A$ satisfies (p-type). By choice of $\Psi(x), \Psi^{\prime}(x)$ and the independence of truth at a pointed model from the choice of a single free variable in a formula we obviously have $t A u$, so that (elem) is satisfied, too.

As for (p-base), since the degree of any atomic formula is 0 , and the above condition implies that $k-m+2 \geq 2$, it is evident that for any $\left(\bar{a}_{m}, a\right) A\left(\bar{b}_{m}, b\right)$ and any unary predicate letter $P \in \Sigma_{\varphi}$ we have $a \models_{i} P(x) \Rightarrow b \models_{j} P(x)$.

To verify condition (p-step), take any $\left(\bar{a}_{m}, a\right) A\left(\bar{b}_{m}, b\right)$ such that $m<k$ and any $d \in U_{j}$ such that $b R_{j} d$. In this case we will also have $m+1 \leq k$.

Then consider the set $\Omega$ of all $\left(\Sigma_{\varphi}, x, k+1-m\right)$-formulas and divide it into the following two subsets:

$$
\begin{aligned}
& \Gamma=\left\{S T(I, x) \in \Omega \mid d \models_{j} S T(I, x)\right\} \\
& \Delta=\left\{S T(I, x) \in \Omega \mid d \forall_{j} S T(I, x)\right\} .
\end{aligned}
$$

These sets are nonempty, since by our assumption we have $k+1-m \geq 1$. Therefore, as we have $r(S T(\perp, x))=0$ and $r(S T(\perp \rightarrow \perp, x))=1$, we will also have $S T(\perp, x) \in \Delta$ and $S T(\perp \rightarrow \perp, x) \in \Gamma$. Then, according to our Lemma 3.7, there are finite nonempty sets of logical equivalents for both $\Gamma$ and $\Delta$. Choosing these finite sets, we in fact choose 
some finite $\left\{S T\left(I_{1}, x\right) \ldots S T\left(I_{n}, x\right)\right\} \subseteq \Gamma,\left\{S T\left(J_{1}, x\right) \ldots S T\left(J_{q}, x\right)\right\} \subseteq \Delta$ such that

$$
\begin{aligned}
& \forall \psi(x) \in \Gamma\left(S T\left(I_{1}, x\right) \wedge \ldots \wedge S T\left(I_{n}, x\right) \models \psi(x)\right) ; \\
& \forall \chi(x) \in \Delta\left(\chi(x) \models S T\left(J_{1}, x\right) \vee \ldots \vee S T\left(J_{q}, x\right)\right) .
\end{aligned}
$$

But then we obtain that

$$
b \not{ }_{j} S T\left(\left(I_{1} \wedge \ldots \wedge I_{n}\right) \rightarrow\left(J_{1} \vee \ldots \vee J_{q}\right), x\right) .
$$

In fact, $d$ falsifies this implication for $\left(M_{j}, b\right)$. But every formula in both sets

$$
\left\{S T\left(I_{1}, x\right) \ldots S T\left(I_{n}, x\right)\right\}, \quad\left\{S T\left(J_{1}, x\right) \ldots S T\left(J_{q}, x\right)\right\}
$$

is, by their choice, a $\left(\Sigma_{\varphi}, x, k+1-m\right)$-formula, and so the standard translation of implication under consideration must be a $\left(\Sigma_{\varphi}, x, k+2-m\right)$-formula. Note, further, that by $\left(\bar{a}_{m}, a\right) A\left(\bar{b}_{m}, b\right)$ we have

$$
\left(M_{i}, a\right) \leq_{k-m+2}\left(M_{j}, b\right)
$$

and therefore this implication must be false at $\left(M_{i}, a\right)$ as well. But then take any $c \in U_{i}$ such that $a R_{i} c$ and $c$ verifies the conjunction in the antecedent of the formula but falsifies its consequent. We must conclude then, by the choice of $\left\{S T\left(I_{1}, x\right) \ldots S T\left(I_{n}, x\right)\right\}$, that $c \models{ }_{i} \Gamma$ and so, by the definition of $A$, and given that $m+1 \leq k$, that

$$
\left(\bar{b}_{m}, b, d\right) A\left(\bar{a}_{m}, a, c\right) .
$$

Since, in addition, $c$ falsifies every formula from $\left\{S T\left(J_{1}, x\right) \ldots S T\left(J_{q}, x\right)\right\}$, then, by the choice of this set, we must conclude that every $\left(\Sigma_{\varphi}, x, k+1-m\right)$-formula that is a standard $x$-translation of an intuitionistic formula false at $\left(M_{j}, d\right)$ is also false at $\left(M_{i}, c\right)$. But then, again by the definition of $A$, and given the fact that $m+1 \leq k$, we must also have $\left(\bar{a}_{m}, a, c\right) A\left(\bar{b}_{m}, b, d\right)$, and so condition (p-step) holds.

Therefore $A$ is an $\left\langle\left(M_{1}, t\right),\left(M_{2}, u\right)\right\rangle_{k}$-asimulation, and we have got our contradiction in place.

The parametrized version of our main result is now easily derived:

THEOREM 3.12. A formula $\varphi(x)$ is equivalent to a standard $x$-translation of an intuitionistic formula iff there exists a $k \in \mathbb{N}$ such that $\varphi(x)$ is invariant with respect to $k$-asimulations.

Proof. Let $\varphi(x)$ be equivalent to $S T(I, x)$. Then by Corollary 3.6, $S T(I, x)$ is invariant with respect to $r(S T(I, x))$-asimulations, and, therefore, so is $\varphi(x)$. In the other direction, let $\varphi(x)$ be invariant with respect to $k$-asimulations for some $k$. If $k \leq r(\varphi)$, then every $r(\varphi)$-asimulation is a $k$-asimulation, so $\varphi(x)$ is invariant with respect to $r(\varphi)$-asimulations and hence, by Theorem 3.11, $\varphi(x)$ is equivalent to a standard $x$-translation of an intuitionistic formula. If, on the other hand, $r(\varphi)<k$, then set $l:=k-r(\varphi)$ and consider variables $\bar{y}_{l}$ not occurring in $\varphi(x)$. Then $r\left(\forall \bar{y}_{l} \varphi(x)\right)=k$ and $\varphi(x)$ is logically equivalent to $\forall \bar{y}_{l} \varphi(x)$, so the latter formula is also invariant with respect to $k$-asimulations, and hence by Theorem $3.11 \forall \bar{y}_{l} \varphi(x)$ is logically equivalent to a standard $x$-translation of an intuitionistic formula. But then $\varphi(x)$ is equivalent to this standard $x$-translation as well.

Although Theorem 3.12 is considerably weaker than Theorem 4.15, which is our main result, it is worth noting that it still gives an operational criterion of equivalence of a given first-order formula to a standard translation of an intuitionistic formula. Moreover, the 
above proof of Theorem 3.11 looks somewhat more 'constructive' than the corresponding part of proof of Theorem 4.15 as it gives an explicit, if not necessarily effective, representation of an intuitionistic formula $I$ such that a given $\varphi(x)$ is logically equivalent to $S T(I, x)$, which might be an advantage from an intuitionistic viewpoint.

§4. The main result. We begin with a formal definition of the notion of unparametrized asimulation. The basic idea is the same as with $k$-asimulation, but paths and bounds are done away with:

Definition 4.1. Let $\left(M_{1}, t\right),\left(M_{2}, u\right)$ be two pointed $\Theta$-models. A binary relation $A$ is called $\left\langle\left(M_{1}, t\right),\left(M_{2}, u\right)\right\rangle$-asimulation iff for any $i, j \in\{1,2\}$, any $a \in U_{i}, b, d \in U_{j}$, any unary predicate letter $P \in \Theta$ the following conditions hold:

$$
\begin{aligned}
& A \subseteq\left(U_{1} \times U_{2}\right) \cup\left(U_{2} \times U_{1}\right) \\
& t A u \\
& \left.\left(a A b \wedge a \models_{i} P(x)\right) \Rightarrow b \models_{j} P(x)\right) \\
& \left(a A b \wedge b R_{j} d\right) \Rightarrow \exists c \in U_{i}\left(a R_{i} c \wedge c \overleftrightarrow{A} d\right) .
\end{aligned}
$$

EXAMPle 4.2. Consider again the models $M_{1}$ and $M_{2}$ from Example 3.2. The binary relation $B=\{(a, d),(b, e),(e, b)\}$ is an $\left\langle\left(M_{1}, a\right),\left(M_{2}, d\right)\right\rangle$-asimulation.

Relation $B$ from the previous example has an obvious connection with relation $A$ from Example 3.2. This observation can be generalized: it turns out that one can transform an asimulation into a $k$-asimulation for every $k \in \mathbb{N}$ and vice versa:

DEFINITION 4.3. Let $A$ and $B$ be binary relations such that for some sets $S$ and $T$,

$$
\begin{gathered}
A \subseteq(S \times T) \cup(T \times S) ; \\
B \subseteq \bigcup_{n>0}\left(\left(S^{n} \times T^{n}\right) \cup\left(T^{n} \times S^{n}\right)\right) .
\end{gathered}
$$

Then we define relations $A \uparrow$ and $B \downarrow$ in the following way:

$$
\begin{gathered}
A \uparrow=\left\{\left\langle\left(\bar{c}_{n}, c\right),\left(\bar{d}_{n}, d\right)\right\rangle \in\left(S^{n+1} \times T^{n+1}\right) \cup\left(T^{n+1} \times S^{n+1}\right) \mid c A d\right\} ; \\
B \downarrow=\left\{\langle c, d\rangle \mid \exists \bar{c}_{n} \bar{d}_{n}\left(\left(\bar{c}_{n}, c\right) B\left(\bar{d}_{n}, d\right)\right)\right\} .
\end{gathered}
$$

LemmA 4.4. Let $\left(M_{1}, t\right),\left(M_{2}, u\right)$ be two pointed $\Theta$-models, A a binary relation. Then:

1. If $A$ is an $\left\langle\left(M_{1}, t\right),\left(M_{2}, u\right)\right\rangle$-asimulation, then $A \uparrow$ is an $\left\langle\left(M_{1}, t\right),\left(M_{2}, u\right)\right\rangle_{k}$-asimulation for all $k \in \mathbb{N}$.

2. If $A$ is an $\left\langle\left(M_{1}, t\right),\left(M_{2}, u\right)\right\rangle_{k}$-asimulation for all $k \in \mathbb{N}$, then $A \downarrow$ is an $\left\langle\left(M_{1}, t\right),\left(M_{2}, u\right)\right\rangle$-asimulation.

Proof. (1) $A \uparrow$ clearly satisfies (p-type), and we obviously have $t A \uparrow u$. Since for any $i, j \in\{1,2\}$, and any $\left(\bar{c}_{n}, c\right)$ in $U_{i}^{n+1},\left(\bar{d}_{n}, d\right)$ in $U_{j}^{n+1}$ such that $\left(\bar{c}_{n}, c\right) A \uparrow\left(\bar{d}_{n}, d\right)$ we have $c A d$, condition (p-base) for $A \uparrow$ follows from the fulfilment of condition (base) for $A$. Also, if $\left(\bar{a}_{n}, a\right) A \uparrow\left(\bar{b}_{n}, b\right)$ then $a A b$, and if, further, $d \in U_{j}$ and $b R_{j} d$, then by condition (step) we can choose $c \in U_{i}$ such that $a R_{i} c, c A d$ and $d A c$. But then, by definition of $A \uparrow$ we will also have $\left(\bar{a}_{n}, a, c\right) A \uparrow\left(\bar{b}_{n}, b, d\right)$ and $\left(\bar{b}_{n}, b, d\right) A \uparrow\left(\bar{a}_{n}, a, c\right)$ so condition (p-step) for $A \uparrow$ is fulfilled for every $k$. 
(2) We only consider condition (step) as the fulfilment of the other conditions is clear. Assume that $i, j \in\{1,2\}, a \in U_{i}, b, d \in U_{j}, b R_{j} d$, and $a A \downarrow b$. Then for some $\bar{a}_{n} \in U_{i}$ and some $\bar{b}_{n} \in U_{j}$ it is true that $\left(\bar{a}_{n}, a\right) A\left(\bar{b}_{n}, b\right)$. Since $A$ is assumed to be $(n+1)$ asimulation, then, by condition (p-step), for some $c \in U_{i}$ we get $\left(\bar{a}_{n}, a, c\right) \stackrel{\leftrightarrow}{A}\left(\bar{b}_{n}, b, d\right)$ and so, by definition of $A \downarrow$, we get both $c A \downarrow d$ and $d A \downarrow c$.

While Lemma 4.4 says that the existence of a single $k$-asimulation for every $k \in \mathbb{N}$ between any two given pointed models implies the existence of asimulation simpliciter, the following example shows that it is not the case that the existence of some $k$-asimulation for every $k \in \mathbb{N}$ between any two given pointed models implies the existence of an asimulation between them.

EXAMPLE 4.5. Consider two $\left\{R^{2}, P^{1}\right\}$-models $M_{1}$ and $M_{2}$ such that $\iota_{1}(P)=\iota_{2}(P)=\varnothing$, $U_{1}=\{a\} \cup\left\{b_{j}^{i} \mid i \leq j, i, j \in \mathbb{N}\right\}, U_{2}=\mathbb{N}, R_{2}=<$, and

$$
x R_{1} y \Leftrightarrow(x=a \wedge y \neq a) \vee\left(x=b_{i}^{j} \wedge y=b_{i}^{k} \wedge j<k\right) .
$$

So $M_{1}$ is a tree where finite branches of arbitrary length spring out from its root a, while $M_{2}$ is a single infinite branch. It is easy to see now that for a given $k \in \mathbb{N}$ the following relation $A_{k}$

$$
\begin{aligned}
\{\langle a, 0\rangle\} \cup\{ & \left\{\left(a, b_{0}^{k-1}, \ldots, b_{j}^{k-1}\right),(0, \ldots, j+1)\right\rangle, \\
& \left.\left\langle(0, \ldots, j+1),\left(a, b_{0}^{k-1}, \ldots, b_{j}^{k-1}\right)\right\rangle \mid j<k\right\}
\end{aligned}
$$

is an $\left\langle\left(M_{1}, a\right),\left(M_{2}, 0\right)\right\rangle_{k}$-asimulation, but there exists no $\left\langle\left(M_{1}, a\right),\left(M_{2}, 0\right)\right\rangle$-asimulation since no branch of $M_{1}$ is long enough to provide counterparts for all <-successors of 0.

EXAMPLE 4.6. Consider again the models $M_{1}$ and $M_{2}$ from Example 3.2. Since the formula

$$
\exists y(R(x, y) \wedge P(y))
$$

is true at $\left(M_{1}, a\right)$, but not at $\left(M_{2}, d\right)$, the fact that binary relation $B$ from Example 4.2 is an $\left\langle\left(M_{1}, a\right),\left(M_{2}, d\right)\right\rangle$-asimulation means that this formula is not invariant with respect to asimulations.

The first part of Lemma 4.4, together with the above results, actually implies the easy part of our main theorem:

COROLlary 4.7. If $\varphi(x)$ is equivalent to a standard $x$-translation of an intuitionistic formula, then $\varphi(x)$ is invariant with respect to asimulations.

Proof. Let $\varphi(x)$ be equivalent to a standard $x$-translation of an intuitionistic formula, let $A$ be an $\left\langle\left(M_{1}, a\right),\left(M_{2}, b\right)\right\rangle$-asimulation. Then by Lemma $4.4 A \uparrow$ is an $\left\langle\left(M_{1}, a\right),\left(M_{2}, b\right)\right\rangle_{k}$-asimulation for every $k$. So if we have $a \models_{1} \varphi(x)$, but not $b \models_{2} \varphi(x)$, then $\varphi(x)$ is not invariant with respect to $k$-asimulations for any $k$, which is in contradiction with Theorem 3.12.

In what follows we will also need some notions and facts from model theory of modal propositional logic. Thus, standard modal $x$-translation $\operatorname{Tr}(m, x)$ of a modal propositional formula $m$ in first-order logic is defined by the following induction on the complexity of 
modal propositional formula:

$$
\begin{aligned}
\operatorname{Tr}\left(p_{n}, x\right) & =P_{n}(x) ; \\
\operatorname{Tr}\left(m \wedge m^{\prime}, x\right) & =\operatorname{Tr}(m, x) \wedge \operatorname{Tr}\left(m^{\prime}, x\right) ; \\
\operatorname{Tr}(\neg m, x) & =\neg \operatorname{Tr}(m, x) ; \\
\operatorname{Tr}(\square m, x) & =\forall y(R(x, y) \rightarrow \operatorname{Tr}(m, y)) .
\end{aligned}
$$

Again, we assume in the last clause that $y$ is fresh with respect to $\operatorname{Tr}(m, x)$.

Another important idea is the notion of bisimulation:

DEFINITION 4.8. Let $\left(M_{1}, t\right),\left(M_{2}, u\right)$ be pointed $\Theta$-models. Then a binary relation $E \subseteq$ $U_{1} \times U_{2}$ is a $\left\langle\left(M_{1}, t\right),\left(M_{2}, u\right)\right\rangle$-bisimulation iff for any $a, c \in M_{1}, b, d \in M_{2}$, and for any unary predicate letter $P \in \Theta$ the following conditions hold:

$$
\begin{array}{lr}
E \subseteq U_{1} \times U_{2} ; & \text { (b-type) } \\
t E u ; & \text { (elem) } \\
a E b \Rightarrow\left(a \models_{1} P(x) \Leftrightarrow b \models_{2} P(x)\right) ; & \text { (b-base) } \\
\left(a E b \wedge a R_{1} c\right) \Rightarrow \exists h \in U_{2}\left(b R_{2} h \wedge c E h\right) ; & \text { (forth) } \\
\left(a E b \wedge b R_{2} d\right) \Rightarrow \exists g \in U_{1}\left(a R_{1} g \wedge g E d\right) . & \text { (back) }
\end{array}
$$

The concept of standard modal translation and that of bisimulation invariance are tied together by van Benthem's famous modal characterization theorem:

THEOREM 4.9. A formula $\varphi(x)$ is invariant with respect to bisimulations iff it is equivalent to a standard modal $x$-translation of a modal propositional formula.

Its proof can be found, for example, in Blackburn et al. (2001, theorem 2.68, pp. 103104). It is easy to see that our main result below (Theorem 4.16) is in an analogy with van Benthem's characterization theorem for intuitionistic propositional logic both in its formulation and in methods of proof employed.

Next lemma establishes a link from asimulation invariance to bisimulation invariance, which we will need in order to use van Benthem's result in proof of our main theorem:

LEMMA 4.10. Let $\varphi(x)$ be a formula invariant with respect to asimulations. Then:

1. $\varphi(x)$ is invariant with respect to bisimulations.

2. $\neg \varphi(x)$ is invariant with respect to bisimulations.

Proof. (1) Let $\Sigma_{\varphi} \subseteq \Theta$, let $\left(M_{1}, t\right),\left(M_{2}, u\right)$ be pointed $\Theta$-models such that $t \models_{1} \varphi(x)$ and let $E$ be an $\left\langle\left(M_{1}, t\right),\left(M_{2}, u\right)\right\rangle$-bisimulation. Then define $A$ as $E \cup E^{-1}$. It is easy to verify that $A$ is an $\left\langle\left(M_{1}, t\right),\left(M_{2}, u\right)\right\rangle$-asimulation: we obviously have $t A u$, and conditions (type), (base) are fulfilled.

To verify (step), assume that $a A b$. Then either $a \in U_{1} \wedge b \in U_{2}$ or $a \in U_{2} \wedge b \in U_{1}$. So in the former case, by Definition 4.8 and our definition of $A$, we must have $a E b$, while in the latter case we must have $b E a$. Therefore, in the former case, if $b R_{2} d$, we apply condition (back) and choose $g \in U_{1}$ such that $a R_{1} g \wedge g E d$, and so, by definition of $A$, we have both $g A d$ and $d A g$. In the latter case, if $b R_{1} d$ we apply condition (forth) and choose $h \in U_{2}$ such that $a R_{2} h \wedge d E h$, and so, again by definition of $A$, we have both $d A h$ and $h A d$. Thus $A$ is an $\left\langle\left(M_{1}, t\right),\left(M_{2}, u\right)\right\rangle$-asimulation and since $\varphi(x)$ is invariant with respect to asimulations, we must have $u \models_{2} \varphi(x)$. The first statement of the lemma is proved. 
(2) Let $\Sigma_{\varphi} \subseteq \Theta$, let $\left(M_{1}, t\right),\left(M_{2}, u\right)$ be pointed $\Theta$-models and let $E$ be an $\left\langle\left(M_{1}, t\right),\left(M_{2}, u\right)\right\rangle$-bisimulation such that $t \models_{1} \neg \varphi(x)$ but $u \models_{2} \neg \varphi(x)$. But then we have that $u \models_{2} \varphi(x)$ and $t \forall_{1} \varphi(x)$. Again, define $A$ as $E \cup E^{-1}$. In the previous paragraph it was established that $A$ verifies conditions (type)-(step). But since $t E u$, we also have $u A t$ and so $A$ is in fact an $\left\langle\left(M_{2}, u\right),\left(M_{1}, t\right)\right\rangle$-asimulation, which contradicts our assumption that $\varphi(x)$ is invariant with respect to asimulations.

A final conceptual ingredient which we will need to get our main result is the notion of modal saturation:

DEFINITION 4.11. A model $M$ is called modally saturated iff for any $a \in U$ and for any set $\Delta(x)$ of standard modal $x$-translations of modal propositional formulas it is true that

$$
\begin{aligned}
& {[\forall(\Gamma(x) \subseteq \Delta(x))(\Gamma(x) \text { is finite } \Rightarrow \exists b \in U(l(R)(a, b) \wedge M, b \models \Gamma(x)))] \Rightarrow} \\
& \Rightarrow \exists c \in U(l(R)(a, c) \wedge M, c \models \Delta(x)) .
\end{aligned}
$$

This notion is important because, as Lemma 4.13 shows, for modally saturated models asimulation in a sense has its intended meaning of inclusion of sets of intuitionistic formulas true at corresponding states.

DEFINITION 4.12. Let $(M, a)$ be a pointed $\Theta$-model. Then we call intuitionistic theory of $(M, a)$, or IT $(M, a)$, the set of all standard translations of intuitionistic formulas true at $(M, a)$.

LEMMA 4.13. Let $M_{1}, M_{2}$ be modally saturated $\Theta$-models and let $I T\left(M_{1}, t\right) \subseteq$ IT $\left(M_{2}, u\right)$. Then the relation

$$
A=\left\{\langle a, b\rangle \mid \exists i, j \in\{1,2\}\left(I T\left(M_{i}, a\right) \subseteq I T\left(M_{j}, b\right)\right)\right\}
$$

is an $\left\langle\left(M_{1}, t\right),\left(M_{2}, u\right)\right\rangle$-asimulation.

Proof. It is obvious that $t A u$, and that condition (type) is satisfied as well. Since for any unary predicate letter $P$ and variable $x$ formula $P(x)$ is a standard $x$-translation of an atomic intuitionistic formula, condition (base) is trivially satisfied for $A$. To verify condition (step), choose any $i, j \in\{1,2\}$, and $a \in U_{i}, b, d \in U_{j}$ such that $I T\left(M_{i}, a\right) \subseteq$ $I T\left(M_{j}, b\right)$ and $b R_{j} d$. Then choose any variable $x$ and let

$$
\Upsilon=\{I \mid S T(I, x) \text { is a } \Theta \text {-formula }\} .
$$

Define the following two subsets of $\Upsilon$ :

$$
\begin{aligned}
& \Gamma=\left\{I \in \Upsilon \mid d \models_{j} S T(I, x)\right\} ; \\
& \Delta=\left\{I \in \Upsilon \mid d \forall_{j} S T(I, x)\right\} .
\end{aligned}
$$

We have by the choice of $\Gamma, \Delta$ that for every finite $\Gamma^{\prime} \subseteq \Gamma$ and $\Delta^{\prime} \subseteq \Delta$ the formula $S T\left(\bigwedge\left(\Gamma^{\prime}\right) \rightarrow \bigvee\left(\Delta^{\prime}\right), x\right)$ is falsified by $d$ for $\left(M_{j}, b\right)$. So, by our premise that $I T\left(M_{i}, a\right) \subseteq I T\left(M_{j}, b\right)$, the standard $x$-translation of every such implication must be false at $\left(M_{i}, a\right)$ as well. This means that every finite subset of the set

$$
\{S T(I, x) \mid I \in \Gamma\} \cup\{\neg S T(I, x) \mid I \in \Delta\}
$$

is true at some $c \in U_{i}$ such that $a R_{i} c$. (We set $\Delta^{\prime}=\{S T(\perp, x)\}$ if the finite set in question has an empty intersection with $\Delta$ and $\Gamma^{\prime}=\{S T(\perp \rightarrow \perp, x)\}$ if it has an empty intersection with $\Gamma$.) But by Corollary 4.7 and Lemma 4.10 every formula in the set under consideration is invariant with respect to bisimulations and hence equivalent to a standard 
modal $x$-translation of a modal propositional formula. Therefore, by modal saturation of both $M_{1}$ and $M_{2}$ there must be a $c \in U_{i}$ such that $a R_{i} c$ and

$$
c \models_{i}\{S T(I, x) \mid I \in \Gamma\} \cup\{\neg S T(I, x) \mid I \in \Delta\} .
$$

By choice of $\Gamma$ and $\Delta$ and by the independence of truth at a pointed model from the choice of a single free variable in a formula we will have both $\operatorname{IT}\left(M_{i}, c\right) \subseteq I T\left(M_{j}, d\right)$ and $I T\left(M_{j}, d\right) \subseteq I T\left(M_{i}, c\right)$ and so condition (step) is also verified.

Another important fact about modally saturated models is that one can always extend a given model to make it modally saturated:

Lemma 4.14. Let $M$ be a $\Theta$-model. Then there is a $\Theta$-model $M^{\prime}$ such that $M^{\prime}$ is modally saturated, and there is a map $f: U \rightarrow U^{\prime}$ such that for any formula $\varphi(x)$ which is invariant with respect to bisimulations and any $a \in U$ it is true that

$$
M, a \models \varphi(x) \Leftrightarrow M^{\prime}, f(a) \models \varphi(x) .
$$

Proof. Let $M^{\prime}$ be an ultrafilter extension of $M$ and let $f(a)$ be the principal ultrafilter generated by $a$ for any $a \in U$. Then our lemma follows from propositions 2.59 and 2.61 of Blackburn et al. (2001, pp. 96-97) and Theorem 4.9.

We are prepared now to state and prove our main result.

THEOREM 4.15. Let $\varphi(x)$ be invariant with respect to asimulations. Then $\varphi(x)$ is equivalent to a standard $x$-translation of an intuitionistic formula.

Proof. We may assume that $\varphi(x)$ is satisfiable, for $\perp$ is clearly invariant with respect to asimulations and $\perp \leftrightarrow S T(\perp, x)$ is a valid formula. In what follows we will write $I C(\varphi(x))$ for the set of $\Sigma_{\varphi}$-formulas that are standard $x$-translations of intuitionistic formulas following from $\varphi(x)$.

Our strategy will be to show that $\operatorname{IC}(\varphi(x)) \models \varphi(x)$. Once this is done we will apply compactness of first-order logic and conclude that $\varphi(x)$ is equivalent to a finite conjunction of standard $x$-translations of intuitionistic formulas and hence to a standard $x$-translation of the corresponding intuitionistic conjunction.

To show this, take any pointed $\Sigma_{\varphi}$-model $\left(M_{1}, t\right)$ such that $t \models_{1} I C(\varphi(x))$. Such a model exists because $\varphi(x)$ is satisfiable and $I C(\varphi(x))$ will be satisfied in any pointed model satisfying $\varphi(x)$. Then we can also choose a pointed $\Sigma_{\varphi}$-model $\left(M_{2}, u\right)$ such that $u \models_{2} \varphi(x)$ and $I T\left(M_{2}, u\right) \subseteq I T\left(M_{1}, t\right)$.

For suppose otherwise. Then for any pointed $\Sigma_{\varphi}$-model $(M, a)$ such that $M, a \models \varphi(x)$ we can choose an intuitionistic formula $I_{(M, a)}$ such that $S T\left(I_{(M, a)}, x\right)$ is a $\Sigma_{\varphi}$-formula true at $(M, a)$ but not at $\left(M_{1}, t\right)$. Then consider the set

$$
S=\{\varphi(x)\} \cup\left\{\neg S T\left(I_{(M, a)}, x\right) \mid M, a \models \varphi(x)\right\}
$$

Let $\left\{\varphi(x), \neg S T\left(I_{1}, x\right) \ldots, \neg S T\left(I_{n}, x\right)\right\}$ be a finite subset of this set. If this set is unsatisfiable, then we must have $\varphi(x) \models S T\left(I_{1}, x\right) \vee \ldots \vee S T\left(I_{n}, x\right)$, but then we will also have $\left(S T\left(I_{1}, x\right) \vee \ldots \vee S T\left(I_{n}, x\right)\right) \in I C(\varphi(x)) \subseteq I T\left(M_{1}, t\right)$, and hence $\left(S T\left(I_{1}, x\right) \vee \ldots \vee\right.$ $\left.S T\left(I_{n}, x\right)\right)$ will be true at $\left(M_{1}, t\right)$. But then at least one of

$$
S T\left(I_{1}, x\right), \ldots, S T\left(I_{n}, x\right)
$$

must also be true at $\left(M_{1}, t\right)$, which contradicts the choice of these formulas. Therefore, every finite subset of $S$ is satisfiable, and by compactness $S$ itself is satisfiable as well. But 
then take any pointed $\Sigma_{\varphi}$-model $\left(M^{\prime}, a^{\prime}\right)$ of $S$ and this will be a model for which we will have both $M^{\prime}, a^{\prime} \models S T\left(I_{\left(M^{\prime}, a^{\prime}\right)}, x\right)$ by choice of $I_{\left(M^{\prime}, a^{\prime}\right)}$ and $M^{\prime}, a^{\prime} \models \neg S T\left(I_{\left(M^{\prime}, a^{\prime}\right)}, x\right)$ by the satisfaction of $S$, a contradiction.

Therefore, we will assume in the following that $\left(M_{1}, t\right),\left(M_{2}, u\right)$ are pointed $\Sigma_{\varphi}$-models, $t \models_{1} I C(\varphi(x)), u \models_{2} \varphi(x)$, and $I T\left(M_{2}, u\right) \subseteq I T\left(M_{1}, t\right)$. Then, according to Lemma 4.14, consider modally saturated $\Sigma_{\varphi}$-models $M^{\prime}, M^{\prime \prime}$ and maps $f: U_{1} \rightarrow U^{\prime}$ and $g$ : $U_{2} \rightarrow U^{\prime \prime}$ such that for any $\Sigma_{\varphi}$-formula $\chi(x)$ which is invariant with respect to bisimulations and for any $a \in U_{1}$ and $b \in U_{2}$ we have

$$
M_{1}, a \models \chi(x) \Leftrightarrow M^{\prime}, f(a) \models \chi(x) ; M_{2}, b \models \chi(x) \Leftrightarrow M^{\prime \prime}, g(b) \models \chi(x)
$$

By our assumption, $\varphi(x)$ is invariant with respect to asimulations and so, by Lemma 4.10 we get:

$$
\begin{aligned}
& M_{1}, t \models \varphi(x) \Leftrightarrow M^{\prime}, f(t) \models \varphi(x) \\
& M^{\prime \prime}, g(u) \models \varphi(x)
\end{aligned}
$$

Any standard $x$-translation of an intuitionistic formula is also, by Corollary 4.7, invariant with respect to asimulations. Therefore, again by Lemma 4.10, we have

$$
I T\left(M^{\prime \prime}, g(u)\right)=I T\left(M_{2}, u\right) \subseteq I T_{\varphi}\left(M_{1}, t\right)=I T\left(M^{\prime}, f(t)\right) .
$$

By modal saturation of $M^{\prime}, M^{\prime \prime}$ and Lemma 4.13 , the relation

$$
A=\left\{\langle a, b\rangle \mid \exists \mu, \mu^{\prime} \in\left\{M^{\prime}, M^{\prime \prime}\right\}\left(I T(\mu, a) \subseteq I T\left(\mu^{\prime}, b\right)\right)\right\}
$$

is an $\left\langle\left(M^{\prime \prime}, g(u)\right),\left(M^{\prime}, f(t)\right)\right\rangle$-asimulation. But then by (36) and asimulation invariance of $\varphi(x)$ we get $M^{\prime}, f(t) \models \varphi(x)$, and further, by (35) we conclude that $M_{1}, a \models \varphi(x)$. Therefore, $\varphi(x)$ in fact follows from $\operatorname{IC}(\varphi(x))$.

The following theorem is an immediate consequence of Corollary 4.7 and Theorem 4.15:

THEOREM 4.16. A formula $\varphi(x)$ is invariant with respect to asimulations iff it is equivalent to a standard $x$-translation of an intuitionistic formula.

Theorem 4.16 stated above establishes a criterion for the equivalence of a first-order formula to a standard translation of intuitionistic formula on arbitrary first-order models. But, unlike in the case of modal propositional logic, some of these models will not be intended models for intuitionistic logic. Therefore it would be interesting to look for the criterion of equivalence of first-order formula to a standard translation of intuitionistic formula over the 'intuitionistic' subclass of first-order models. As the class of intended models of intuitionistic propositional logic constitutes a first-order definable subclass of first-order models in general, we can show that such a criterion is provided by invariance with respect to asimulations on the models from this subclass using but a slight modification of our proof for Theorems 4.15 and 4.16.

To tighten up on terminology, we introduce the following definitions:

DEFINITION 4.17. A $\Theta$-model $M$ is said to be intuitionistic, iff $l(R)$ is transitive and reflexive, and for any unary predicate letter $P \in \Theta$ it is true that

$$
\forall(a, b \in U)(l(R)(a, b) \wedge M, a \models P(x) \Rightarrow M, b \models P(x)) .
$$

The notion of intuitionistic model naturally leads to the following semantic definitions: 
DEFINITION 4.18. $\quad$ 1. $\Gamma$ is intuitionistically satisfiable iff $\Gamma$ is satisfied in some intuitionistic model.

2. $\varphi$ is a consequence of $\Gamma(\Gamma \| \Vdash \varphi)$ over the class of intuitionistic models iff $\Gamma \cup\{\neg \varphi\}$ is intuitionistically unsatisfiable.

3. $\varphi$ is equivalent to $\psi$ over the class of intuitionistic models iff both $\psi \| \vdash \varphi$ and $\varphi \| \vdash$.

Let $\operatorname{Int}(\Theta)$ be the following set of formulas

$$
\begin{aligned}
\{\forall y R(y, y), \forall y z w & ((R(y, z) \wedge R(z, w)) \rightarrow R(y, w))\} \cup \\
\cup & \{\forall y z((P(y) \wedge R(y, z)) \rightarrow P(z)) \mid P \in \Theta\} .
\end{aligned}
$$

It is clear that for any set $\Gamma$ of $\Theta$-formulas and for any $\Theta$-formula $\varphi, \Gamma$ is intuitionistically satisfiable iff $\Gamma \cup \operatorname{Int}(\Theta)$ is satisfiable, and $\Gamma \| \vdash$ iff $\Gamma \cup \operatorname{Int}(\Theta) \models \varphi$.

We say, further, that a formula $\varphi(x)$ is intuitionistically invariant with respect to asimulations iff it is invariant with respect to the class of asimulations connecting intuitionistic models.

EXAMPLE 4.19. Formula $\exists y(R(x, y) \wedge P(y))$ is not intuitionistically invariant with respect to asimulations. However, our argument from Example 4.6 does not show this, because the models considered in this example are not intuitionistic. To prove the absence of intuitionistic invariance with respect to asimulations, consider two $\left\{R^{2}, P^{1}\right\}$-models $M_{2}$ and $M_{3}$ such that $U_{2}=\{a, b, c\}, R_{2}=\{(a, a),(a, b),(a, c),(b, b),(c, c)\}, \iota_{2}(P)=$ $\{c\}$, and $U_{3}=\{d, e\}, R_{3}=\{(d, d),(d, e),(e, e)\}, \iota_{3}(P)=\varnothing$. These are intuitionistic models. Then binary relation $C=\{(a, d),(b, d),(d, b),(b, e),(e, b)\}$ is an $\left\langle\left(M_{2}, a\right)\right.$, $\left.\left(M_{3}, d\right)\right\rangle_{k}$-asimulation. It remains to note that the formula under consideration is true at $\left(M_{2}, a\right)$ but not at $\left(M_{3}, d\right)$.

Now for the criterion of equivalence on the restricted class of intuitionistic models:

THEOREM 4.20. Let $\varphi(x)$ be intuitionistically invariant with respect to asimulations. Then $\varphi(x)$ is equivalent over the class of intuitionistic models to a standard $x$-translation of an intuitionistic formula.

Proof. We may assume that $\varphi(x)$ is intuitionistically satisfiable, otherwise $\varphi(x)$ is equivalent to $S T(\perp, x)$ over the class of intuitionistic models and we are done. In what follows we will write $\operatorname{Int} C(\varphi(x))$ for the set of $\Sigma_{\varphi}$-formulas that are standard $x$-translations of intuitionistic formulas following from $\varphi(x)$ over the class of intuitionistic models.

Our strategy will be to show that $\operatorname{Int} C(\varphi(x)) \| \Vdash \varphi(x)$. Once this is done we will conclude that

$$
\operatorname{Int}\left(\Sigma_{\varphi}\right) \cup \operatorname{Int} C(\varphi(x)) \models \varphi(x) .
$$

Then we apply compactness of first-order logic and conclude that $\varphi(x)$ is equivalent to a finite conjunction $\bigwedge \Psi(x)$ of formulas from this set. But it follows then that $\varphi(x)$ is equivalent to the conjunction of the set $\operatorname{Int} C(\varphi(x)) \cap \Psi(x)$ over the class of intuitionistic models. In fact, by our choice of $\operatorname{Int} C(\varphi(x))$ we have

$$
\varphi(x) \Vdash \uparrow(\operatorname{Int} C(\varphi(x)) \cap \Psi(x)),
$$

And by our choice of $\Psi(x)$ we have

$$
\operatorname{Int}\left(\Sigma_{\varphi}\right) \cup(\operatorname{Int} C(\varphi(x)) \cap \Psi(x)) \models \varphi(x)
$$


and hence

$$
\operatorname{Int} C(\varphi(x)) \cap \Psi(x) \Vdash \mapsto \varphi(x) .
$$

To show that $\operatorname{Int} C(\varphi(x)) \| \vdash \varphi(x)$, take any pointed intuitionistic $\Sigma_{\varphi}$-model $\left(M_{1}, t\right)$ such that $t \models_{1} \operatorname{Int} C(\varphi(x))$. Such a model exists, because $\varphi(x)$ is intuitionistically satisfiable and $\operatorname{Int} C(\varphi(x))$ will be intuitionistically satisfied in any pointed intuitionistic model satisfying $\varphi(x)$. Then we can also choose a pointed intuitionistic $\Sigma_{\varphi}$-model $\left(M_{2}, u\right)$ such that $u \models_{2} \varphi(x)$ and $I T\left(M_{2}, u\right) \subseteq I T\left(M_{1}, t\right)$.

For suppose otherwise. Then for any pointed intuitionistic $\Sigma_{\varphi}$-model $(M, a)$ such that $M, a \models \varphi(x)$ we can choose an intuitionistic formula $I_{(M, a)}$ such that $\operatorname{ST}\left(I_{(M, a)}, x\right)$ is a $\Sigma_{\varphi}$-formula true at $(M, a)$ but not at $\left(M_{1}, t\right)$. Then consider the set

$$
S=\{\varphi(x)\} \cup\left\{\neg S T\left(I_{(M, a)}, x\right) \mid M \text { is intuitionistic, } M, a \models \varphi(x)\right\}
$$

Let $\left\{\varphi(x), \neg S T\left(I_{1}, x\right) \ldots, \neg S T\left(I_{n}, x\right)\right\}$ be a finite subset of this set. If this set is intuitionistically unsatisfiable, then we must have

$$
\varphi(x) \| \vdash S T\left(I_{1}, x\right) \vee \ldots \vee S T\left(I_{n}, x\right),
$$

but then we will also have

$$
\left(S T\left(I_{1}, x\right) \vee \ldots \vee S T\left(I_{n}, x\right)\right) \in \operatorname{Int} C(\varphi(x)) \subseteq I T\left(M_{1}, t\right),
$$

and hence $\left(S T\left(I_{1}, x\right) \vee \ldots \vee S T\left(I_{n}, x\right)\right)$ will be true at $\left(M_{1}, t\right)$. But then at least one of $S T\left(I_{1}, x\right) \ldots, S T\left(I_{n}, x\right)$ must also be true at $\left(M_{1}, t\right)$, which contradicts the choice of these formulas. Therefore, every finite subset of $S$ is intuitionistically satisfiable. But then every finite subset of the set $S \cup \operatorname{Int}\left(\Sigma_{\varphi}\right)$ is satisfiable as well. By compactness of first-order $\operatorname{logic} S \cup \operatorname{Int}\left(\Sigma_{\varphi}\right)$ is satisfiable, hence $S$ is satisfiable intuitionistically. But then take any pointed intuitionistic $\Sigma_{\varphi}$-model $\left(M^{\prime}, a^{\prime}\right)$ of $S$ and this will be a model for which we will have both $M^{\prime}, a^{\prime} \models S T\left(I_{\left(M^{\prime}, a^{\prime}\right)}, x\right)$ by choice of $I_{\left(M^{\prime}, a^{\prime}\right)}$ and $M^{\prime}, a^{\prime} \models \neg S T\left(I_{\left(M^{\prime}, a^{\prime}\right)}, x\right)$ by the satisfaction of $S$, a contradiction.

Therefore, for any given pointed intuitionistic $\Sigma_{\varphi}$-model $\left(M_{1}, t\right)$ of $\operatorname{Int} C(\varphi(x))$ we can choose a pointed intuitionistic $\Sigma_{\varphi}$-model $\left(M_{2}, u\right)$ such that $u \models_{2} \varphi(x)$ and $I T\left(M_{2}, u\right) \subseteq$ $I T\left(M_{1}, t\right)$. Then, reasoning exactly as in the proof of Theorem 4.15 , we conclude that $t \models_{1} \varphi(x)$. Therefore, $\varphi(x)$ in fact follows from $\operatorname{Int} C(\varphi(x))$ over the class of intuitionistic models.

THEOREM 4.21. A formula $\varphi(x)$ is intuitionistically invariant with respect to asimulations iff it is equivalent to a standard $x$-translation of an intuitionistic formula over the class of intuitionistic models.

Proof. From left to right our theorem follows from Theorem 4.20. In the other direction, assume that $\varphi(x)$ is equivalent to $S T(I, x)$ over the class of intuitionistic models and assume that for some $\Theta$ such that $\Sigma_{\varphi} \subseteq \Theta$, some pointed intuitionistic $\Theta$-models $\left(M_{1}, t\right)$ and $\left(M_{2}, u\right)$, and some $\left\langle\left(M_{1}, t\right),\left(M_{2}, u\right)\right\rangle$-asimulation $A$ we have $t \models_{1} \varphi(x)$. Then, since $M_{1}$ is intuitionistic, we have $t \models_{1} S T(I, x)$, and, by Corollary 4.7, we have $u \models_{2} S T(I, x)$, but since $S T(I, x)$ is equivalent to $\varphi(x)$ over the class of intuitionistic models and $M_{2}$ is an intuitionistic model, we also have $u \models_{2} \varphi(x)$. Therefore, $\varphi(x)$ is intuitionistically invariant with respect to asimulations.

\$5. Conclusion and further results. Theorems 3.12, 4.16, and 4.21 proved above show that the general idea of asimulation for intuitionistic propositional logic is a faithful 
analogue of the idea of bisimulation for modal propositional logic in many important respects.

A natural further step would be to extend the above results onto the level of intuitionistic predicate logic in order to obtain theorems analogous to theorem 21 of van Benthem (2010, p. 124). In fact, we have already obtained proofs of both parametrized and unparametrized versions of our main result for intuitionistic first-order predicate logic by extending techniques employed above to cover this more general case. We hope to publish these results in some of our future papers.

§6. Acknowledgments. I am grateful to Prof. Grigori Mints (Stanford University), who informed me about the open problem which is solved in this paper, and spent a lot of his time discussing the possible solutions with me. I am grateful to Prof. Balder ten Cate (University of California, Santa Cruz), who made many valuable suggestions on improving the presentation of this result and developing its formulation, both in person and in e-mail correspondence. I would like to thank all the participants of mathematical logic seminar at Stanford University for their attention to my talk based on an earlier version of this paper, and for the discussion that followed. I would like to thank the anonymous referee, who suggested some improvements concerning the presentation of the results, especially examples and definitions.

\title{
BIBLIOGRAPHY
}

Blackburn, P., De Rijke, M., \& Venema, Y. (2001). Modal Logic. Cambridge University Press. Cambridge, UK.

Ebbinghaus, H.-D., Flum, J., \& Thomas, W. (1984). Mathematical Logic (first edition). Springer. New York, NY.

van Benthem, J. (2010). Modal Logic for Open Minds. CSLI Publications. Stanford.

Visser, A., van Benthem, J., de Jongh, D., \& Renardel de Lavalette, G. R. (1995). NNIL, a study in intuitionistic propositional logic. In Ponse, A., de Rijke, M., and Venemapp, Y., editors. Modal Logic and Process Algebra (Amsterdam, 1994), Vol. 53 of CSLI Lecture Notes. Stanford, CA: CSLI Publications, pp. 289-326.

\author{
DEPARTMENT OF PHILOSOPHY \\ URAL FEDERAL UNIVERSITY \\ 51 LENIN AVENUE, OFF 332 \\ YEKATERINBURG \\ RUSSIA 620083 \\ E-mail: grigory.olkhovikov@usu.ru, grigory.olkhovikov@gmail.com
}


Copyright of Review of Symbolic Logic is the property of Cambridge University Press and its content may not be copied or emailed to multiple sites or posted to a listserv without the copyright holder's express written permission. However, users may print, download, or email articles for individual use. 